\title{
Improving the Performance of a Wireless Presentation System
}

\author{
Gin-Xian Kok, Khong Neng Choong, Danial Naghshbandi, and Mohammad Hilmi Mohd Shariff
}

\begin{abstract}
In this paper, we study the performance of a wireless presentation system in a scenario called webviewer. In this scenario, the presenter streams the desktop screen of his/her computing device, typically a laptop, to the wireless presentation box, and the wireless presentation box performs a snapshot of the video stream, encodes, and sends the captured image to a list of subscribers called webviewer clients, periodically. We propose an image data transfer rate throttling scheme and compared it with several other schemes. Simulation study showed that the proposed scheme is able to better utilize the available system resource to improve the satisfaction level of the users in a desktop mirroring session.
\end{abstract}

Index Terms-Desktop mirroring, image distribution, optimal throttling.

\section{INTRODUCTION}

Presentations are held in the academia and corporate world to convey information to a group of people. Presentations are typically conducted using laptops and are displayed on largescreen displays (projectors, televisions, etc.). Traditionally, presentation videos are sent from the laptops to the displays directly using some display cable such as Video Graphics Array (VGA), Digital Visual Interface (DVI), and High Definition Multimedia Interface (HDMI). Due to the rapid advancements in wireless technologies, and video encoding technologies such as H.264 [1] and H.265 [2], it is now possible to stream video from one device to another with high definition and low delay through a wireless connection. The benefits of transferring presentation video wirelessly include reduced clutter, higher mobility freedom, enhanced control, reduced downtime, and lower costs. Fig. 1 shows an example wireless presentation system.

In Fig. 1, the wireless presentation box is connected to the projector via a display cable. The wireless presentation box also acts as a Wi-Fi access point, providing wireless interconnectivity to the laptops that are connected to it. By using custom software downloaded from the wireless presentation box, a user of the system would be able to mirror the contents of his/her laptop's desktop screen onto the large-screen display.

Manuscript received October 9, 2016; revised January 13, 2017.

Gin-Xian KOK is with Wireless Innovation Lab, MIMOS Berhad, Kuala Lumpur, Malaysia (email: gx.kok@mimos.my).

Khong Neng CHOONG, Danial NAGHSHBANDI, and Mohammad Hilmi MOHD SHARIFF are with Wireless Innovation Lab, MIMOS Berhad, Kuala Lumpur, Malaysia(Email: \{kn.choong, danial.naghshbandi, hilmi.shariff\}@mimos.my).

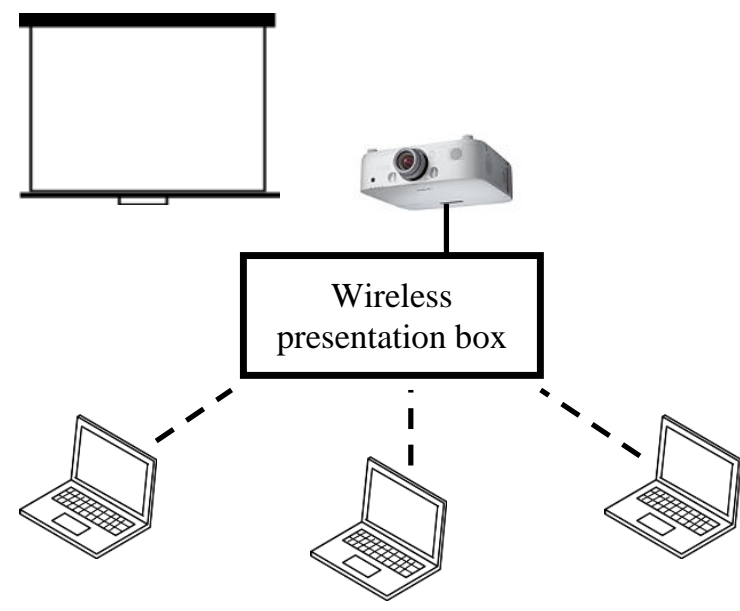

Fig. 1. A wireless presentation system.

While the scenario depicted in Fig. 1 is suitable for a small room, its shortcomings quickly become evident when used in a larger setting such as a lecture hall or an auditorium. In such a scenario, a participant of a meeting may be seated far away from the display and thus may not be able to see the presented video clearly. To overcome this problem, some wireless presentation systems [3], [4] contain a feature called “webviewer". In this feature, the wireless presentation box captures a snapshot of the presentation video at certain times and hosts the snapshots on a HTTP server hosted within the wireless presentation box. A user seated far away from the display can view the snapshots of the presentation through a web browser from his/her device.

In this paper, we performed simple simulation studies to study the performance of a wireless presentation system when used in the webviewer scenario, and try to optimize the performance of the system. This paper is organized as follows. We provide the details of the proposed image data transfer throttling scheme in Section II. Results and discussion is given in Section III. Finally, we conclude in Section IV.

\section{IMAGE DiSTRIBUTTION SCHEMES IN THE WEBVIEWER SCENARIO}

In the webviewer scenario, the presenter streams the desktop screen of his/her computing device to the wireless presentation box. At the same time, the wireless presentation box periodically performs a snapshot of the received video and sends the captured images to a list of subscribers called webviewer clients. We denote the set of all webviewer clients as $\boldsymbol{C}$. Images are encoded using the Joint Photographic Expert Group (JPEG) format and transported using the Transport Control Protocol (TCP).

In this paper, we compare the performance of several image distribution schemes. 


\section{A. Scheme 1: Sending Available Image Immediately}

In this scheme, when an image is available to be distributed to the webviewer clients, the wireless presentation box sends the image to all the webviewer clients immediately.

\section{B. Scheme 2: Adding Random Delay Before Sending Image}

In this scheme, when an image is available to be distributed to the webviewer clients, for each of the webviewer clients, the wireless presentation box introduces a random amount of time (up to a maximum value) before sending the image to the webviewer client. The purpose of this scheme is to avoid a surge in the amount of image data that needs to be transferred across the network whenever an image is available for distribution. Instead, images are sent at different times to reduce the negative impact on the transmission of the video stream.

\section{Scheme 3: Manual Throttling of Image Transfer Rate}

As the number of webviewer clients increases, the amount of data that must be transferred across the network increases. This is because at periodic intervals, the wireless presentation box sends an image to each of the webviewer clients. Depending on the available bandwidth, the wireless presentation box may have to throttle the image data transfer rate to reduce the impact to the transmission of the video stream.

\section{The Proposed Scheme: Optimal Throttling of Image Transfer Rate}

If we assume that the wireless presentation box distributes its available image data transfer rate allocation, $i m g \_r t$ equally among all clients $c \in \boldsymbol{C}$, then the delay between the time the wireless presentation box sends an image and the time a webviewer client completely receives an image, $i m g \_d l$ can be computed as follows, where $i m g \_s z$ is the typical size of a distributed image.

$$
i m g_{-} d l=\frac{i m g_{-} s z}{i m g_{-} r t /|\boldsymbol{C}|}
$$

To ensure that images are delivered in a timely manner, we define a metric called image quality, img_quality. This metric maps $i m g \_d l$ to a value in the interval [ $\left[\begin{array}{ll}0 & 1\end{array}\right]$ using the function shown in Fig. 2.

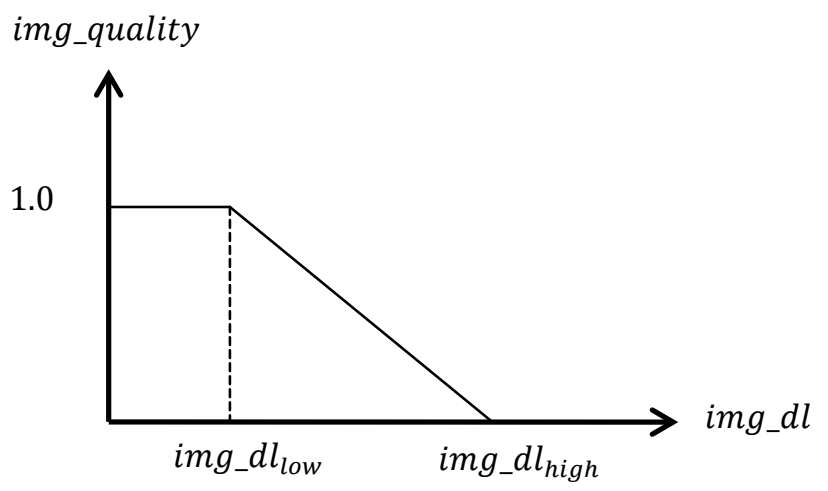

Fig. 2. Image quality as a function of delay.
In Fig. 2, $i m g_{-} d l_{\text {low }}$ and $i m g_{-} d l_{\text {high }}$ are the low and high image delay thresholds, respectively. When the clients receive an image within $i m g_{-} d l_{\text {low }}$, $i m g_{-} q u a l i t y$ is given the value of 1.0. Past $i m g_{-} d l_{\text {low }}$, img_quality decreases as $i m g \_d l$ increases until it finally reaches $i m g_{-} d l_{\text {high }}$. Past $i m g_{-} d l_{\text {high }}$, img_quality is given the value of 0 . The equation for img_quality can be written as follows:

$$
\begin{aligned}
& i m g_{-} \text {quality }=\min \left(\operatorname { m a x } \left(\frac{i m g_{-} d l}{i m g_{-} d l_{l o w}-i m g_{-} d l_{\text {high }}}\right.\right. \\
& \left.\left.+\frac{i m g_{-} d l_{\text {high }}}{i m g_{-} d l_{\text {high }}-i m g_{-} d l_{l o w}}, 0\right), 1.0\right)
\end{aligned}
$$

The video stream is transported using TCP, which ensures reliable data transfer. This means that unless the link between the presenter device and the wireless presentation box fails, there will be no discrepancy between the video stream sent by the presenter device and the video stream received by the wireless presentation box. As a consequence, standard objective video quality metrics such as the Peak Signal to Noise Ratio (PSNR) are not suitable for evaluating video quality in this study. Instead, we define a simple metric called video quality, vid_quality for evaluating video quality. The equation for vid_quality is given as follows, where vid_enc_rt is the data rate of the encoded video bit stream, vid_rt is the actual allocated data rate for the transfer of the video stream in a desktop mirroring session.

$$
\text { vid_quality }=\frac{\min \left(v i d_{-} r t, v i d_{-} e n c_{-} r t\right)}{v i d_{-} e n c_{-} r t}
$$

The video stream of the desktop of the presenter device is captured, encoded, and sent to the wireless presentation box. As the video stream is 'live', the entire video stream of a session is not available prior to the session. Hence, additional allocated rate above vid_rt cannot be utilized to improve video quality and is thus 'wasted'. This explains the min(.) operator used in Eq. 3.

With both image quality and video quality defined, the overall quality of a particular desktop mirroring session, ovr_quality can be defined as follows, where $\alpha$ is the video quality weight.

$$
\begin{aligned}
& \text { ovr_quality }=\alpha(\text { vid_quality }) \\
& +(1-\alpha)(\text { img _ quality })
\end{aligned}
$$

Eq. 4 states that the overall quality of a desktop mirroring session is simply the weighted average of video quality and image quality. It can be observed from Eq. 2 and Eq. 3 that both img_quality and vid_quality take a value in the interval [0 1]. Hence, ovr_quality also takes a value in the same interval. A value of 1.0 for ovr_quality means that the quality of the overall desktop mirroring session is perfect while a value of 0 means that the quality of the overall desktop mirroring session is terrible.

We further define $m a x_{-} n w_{-} t c p_{-} t h r p$ as the maximum achievable TCP throughput in the network. In practice, this 
value can be measured empirically using a tool such as iperf [5], or deduced based on the wireless standard (e.g., IEEE $802.11 \mathrm{~b} / \mathrm{g} / \mathrm{n} / \mathrm{ac}$ ) used in the network. As this maximum throughput is used to send both image and video data, we have the following equation:

$$
\text { vid_rt }+i m g_{-} r t \leq \max { }_{-} n w_{-} t c p_{-} \text {thrp }
$$

Since there is limited available bandwidth, and the bandwidth is used to transfer both video and image data, the wireless presentation box may need to throttle the image data transfer rate as the number of webviewer clients increases so that user satisfaction levels of the presentation participants are maintained at a high level. We would like to determine the optimal image data transfer rate that provides the highest satisfaction level to the presentation participants. This problem can be officially formulated as follows:

$$
i m g_{-} r t^{*}=\underset{i m g_{-} r t}{\operatorname{argmax}}\left(o v r_{-} \text {quality }\right)
$$

s.t.:

A simple algorithm to solve the above problem is given in Fig. 3.

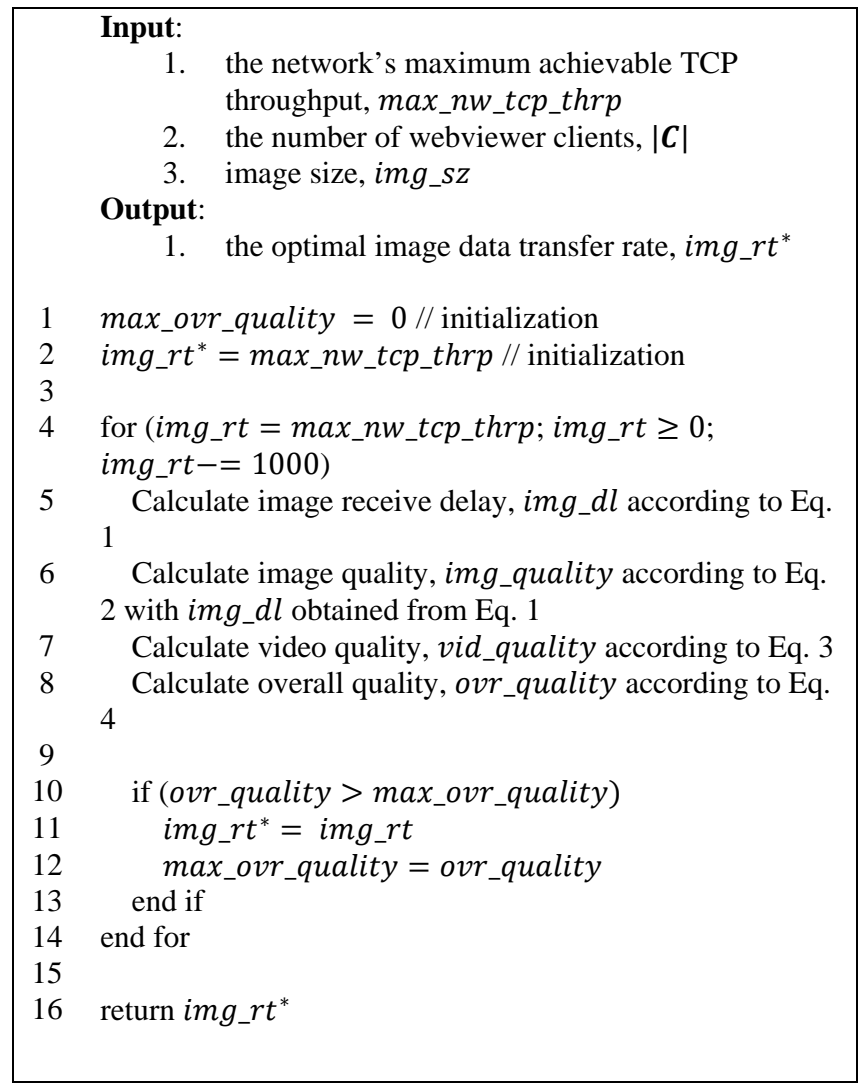

Fig. 3. Algorithm for computing the image throttle rate.

Given the following inputs: (1) the network's maximum achievable TCP throughput, (2) the number of webviewer clients, and (3) image size, the algorithm in Fig. 3 computes the optimal rate for the maximum image data transfer rate.

\section{RESULTS AND DISCUSSION}

We evaluated the various image distribution schemes using a discrete event network simulator called network simulator version 2 (ns-2) [6]. A number of nodes were placed in a square area of dimension $100 \mathrm{~m}$ by $100 \mathrm{~m}$. One of the nodes, i.e., node 0 , was designated as the wireless presentation box and was placed on the coordinate $(50,100)$ in the Cartesian coordinate system. Other nodes are randomly placed within the square area and are connected to node 0 wirelessly. One of the nodes, i.e., node 1 was designated to send a video stream to node 0 using TCP for the entire duration of the simulation, which was $300 \mathrm{~s}$. The bitrate of the video stream was $2 \mathrm{Mbps}$ (or equivalently 250 $\mathrm{kB} / \mathrm{s}$ ). At periodic intervals of $10 \mathrm{~s}$, node 0 sent an image 300 $\mathrm{kB}$ in size to all the webviewer clients using TCP. The MAC and physical layer used is IEEE 802.11 ERP-DSSS. Through experimentation, max_nw_tcp_thrp was determined to be about $500 \mathrm{kB} / \mathrm{s}$. The video quality weight parameter, $\alpha$ was set to 0.7 , while the low and high image delay thresholds, $i m g_{-} d l_{\text {low }}$ and $i m g_{-} d l_{\text {high }}$, were set to $3 \mathrm{~s}$ and $10 \mathrm{~s}$, respectively. The study of the effect of varying these parameters was not carried out and is left as a potential future work. Each scenario was repeated 10 times and the average of the result is used in the our comparisons.

Fig. 4 shows the results from the simulations. For all the image distribution schemes, as the number of webviewer clients increases, video quality (Fig. 4a) and video throughput (Fig. 4e) decrease while image receive delay (Fig. 4b), image packet delay (Fig. 4d), image throughput (Fig. 4f), video packet inter-arrival time (Fig. 4g), and video packet jitter (Fig. 4h) increase. As the number of webviewer clients increases, there is more load within the network as the wireless presentation box has more data to send (it has to send an image to each of the webviewer clients periodically). As the network has a limited amount of bandwidth, when the amount of image data increases, the rate of video data transfer has to be sacrificed. This explains why video quality and video throughput decrease as the number of webviewer clients increases.

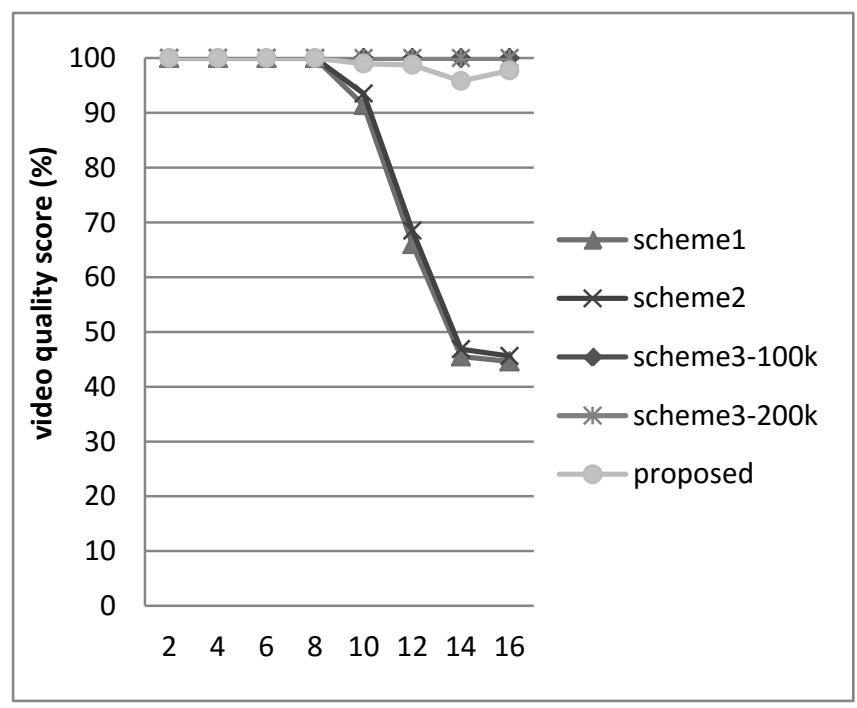

(a) 


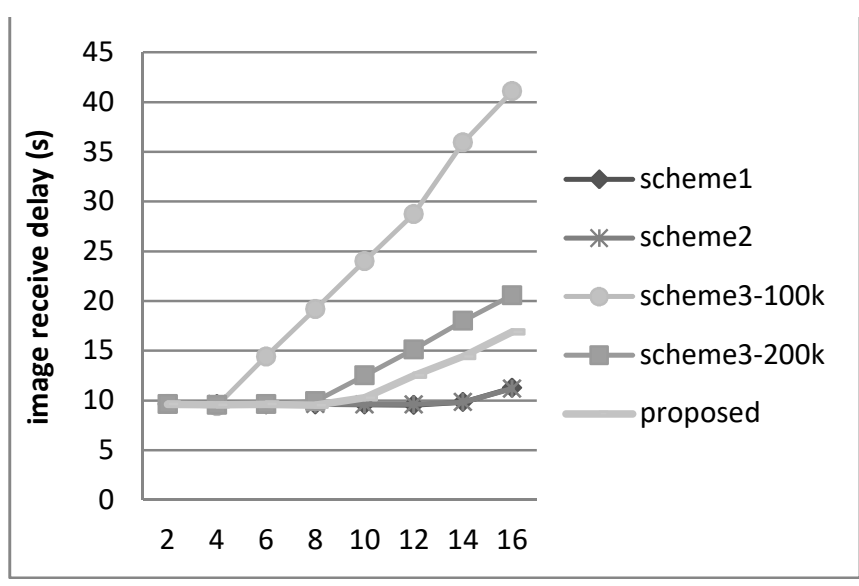

(b)

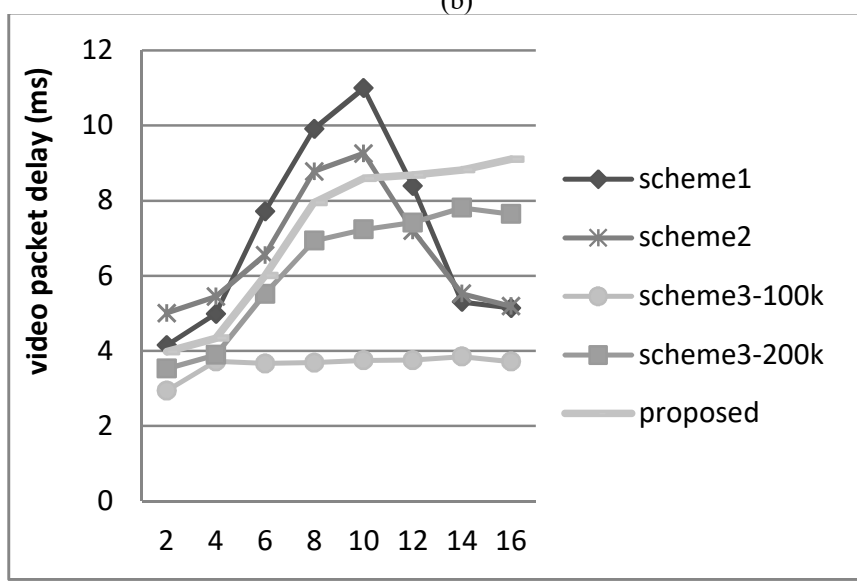

(c)

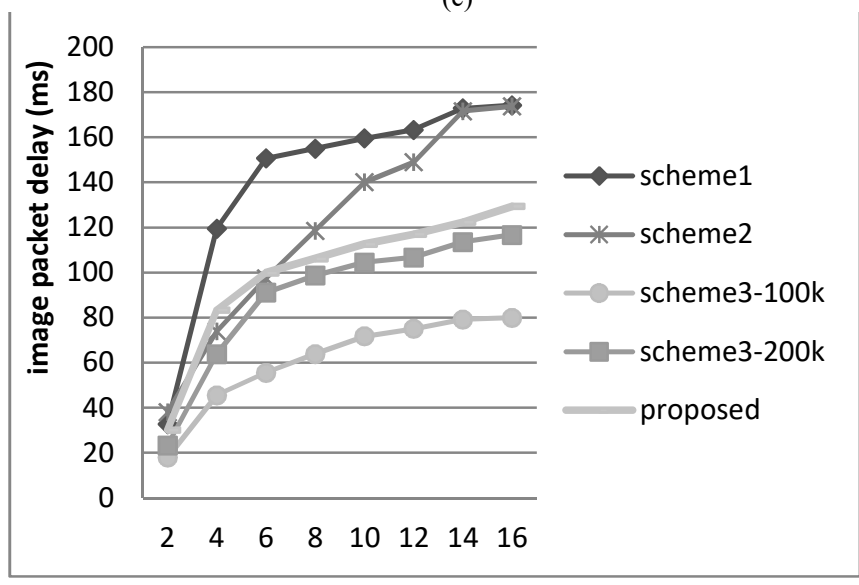

(d)

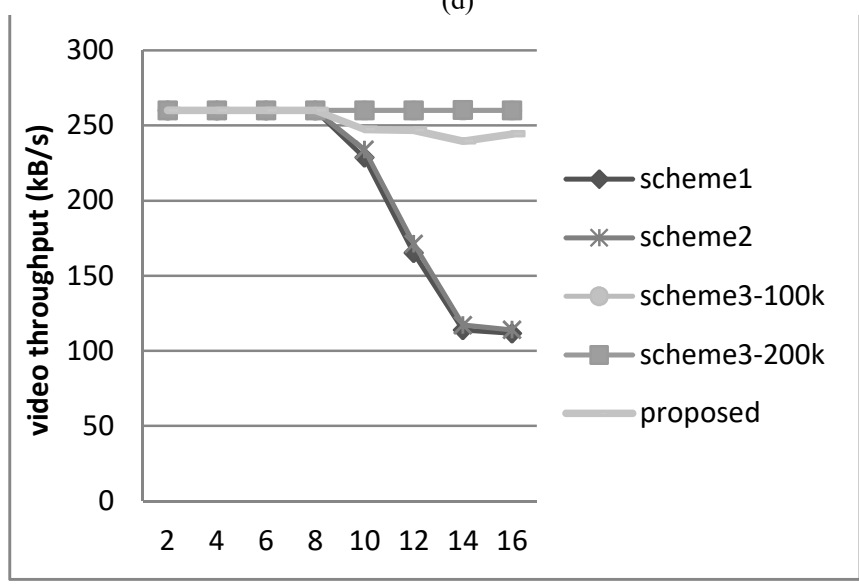

(e)

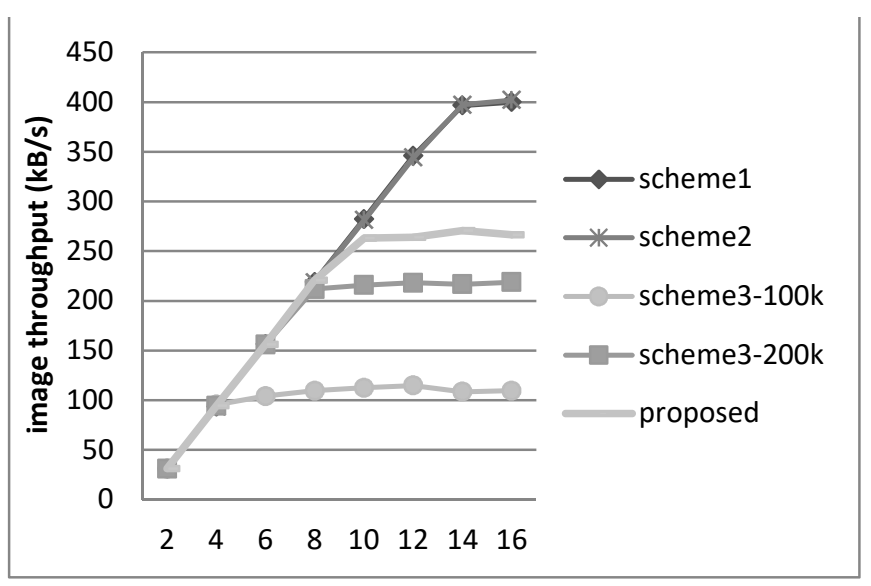

(f)

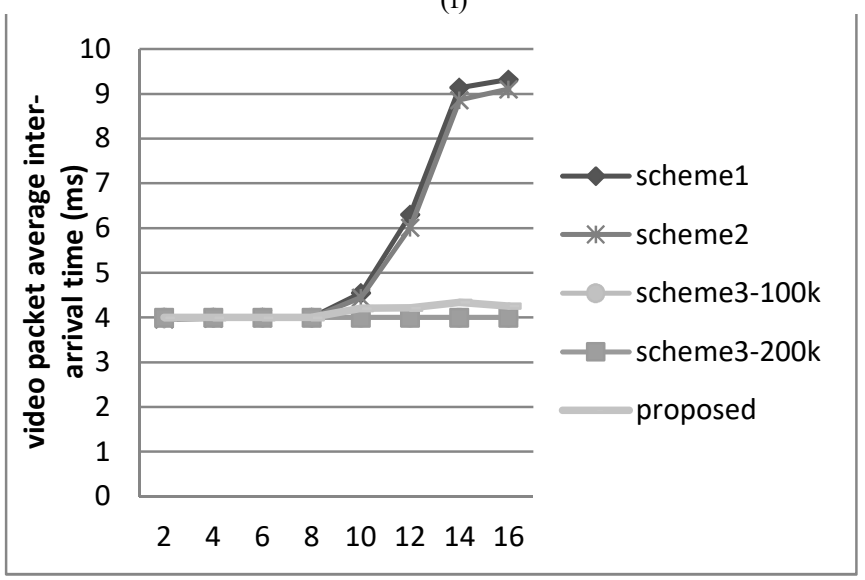

(g)

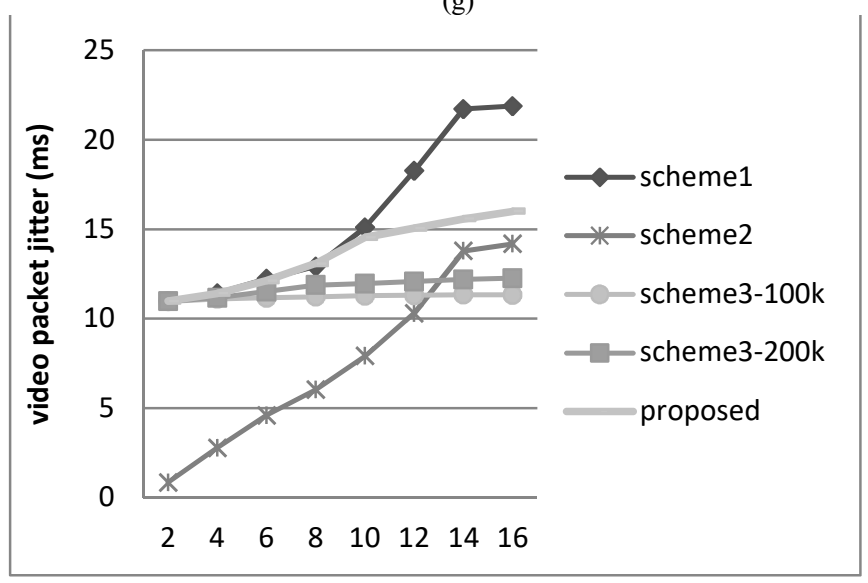

(h)

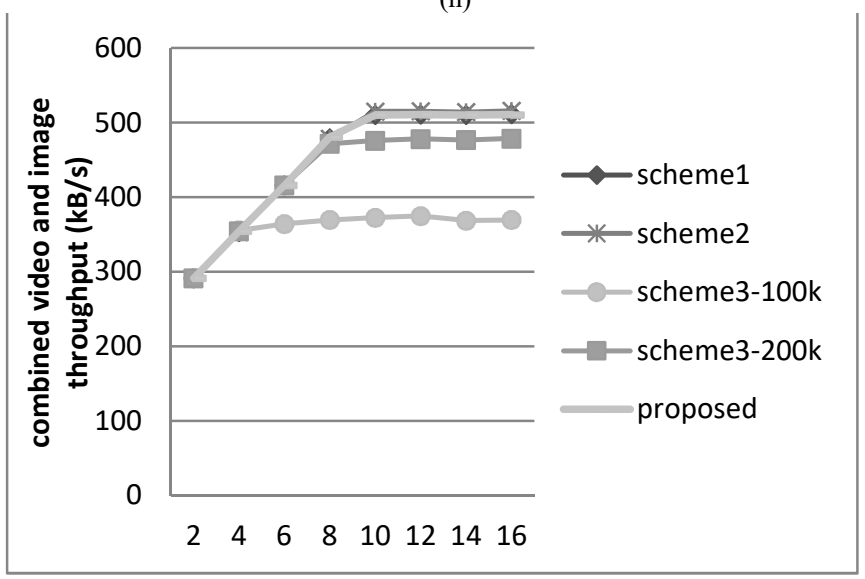

(i)

Fig. 4. (a) Video quality, (b) image receive delay, (c) video packet delay, (d) image packet delay, (e) video throughput, (f) image throughput, (g) video packet inter-arrival time, (h) video packet jitter, (i) combined video and image throughput. 
As can be observed in Figs. $4 \mathrm{a}$ and $4 \mathrm{~b}$, we were unable to observe a significant improvement by sending an image to all the webviewer clients at slightly different times ( scheme2) as opposed to sending the image to all webviewer clients at the same time (scheme1). However, there was some improvement in the video packet delay, image packet delay, video packet inter-arrival time, and video packet jitter as can be observed in Figs. 4c, 4d, 4g, and $4 \mathrm{~h}$, respectively, when images are sent at slightly different times.

The scheme $3-100 k$ and scheme $3-200 k$ schemes implement a maximum image data transfer rate of $100 \mathrm{kB} / \mathrm{s}$ and $200 \mathrm{kB} / \mathrm{s}$, respectively. By throttling image data transfers, we were able to prevent the decrease in video quality as the number of webviewer clients increases, as shown in Fig. 4a (compare scheme $-100 k$, scheme $-200 k$ with scheme 1 and scheme2). However, this came at the expense of increased image receive delay, as can be observed in Fig. 4b. From Fig. 4i, which shows the combined video and image throughput, it can be observed that both scheme 3 $100 k$ and scheme $3-200 k$ were not fully utilizing the maximum achievable TCP rate, which is about $510 \mathrm{kB} / \mathrm{s}$. With the maximum achievable TCP throughput, the number of webviewer clients, and the video rate as inputs, the proposed scheme computes the optimal rate as the maximum image data transfer rate. It can be observed in Fig. 4a that by comparing the proposed scheme with scheme1, the proposed scheme managed to maintain a high video quality level even as the number of webviewer clients is increased. By comparing the proposed, scheme $3-100 k$, and scheme $3-200 k$ schemes, it can be observed that the proposed scheme managed was able to fully utilize the available network resource (Fig. 4i) to reduce image receive delay (Fig. 4b).

\section{CONCLUSIONS}

In this paper, we experimented with various image distribution schemes in the webviewer scenario. In a desktop mirroring session, the presenter streams its desktop screen live to the wireless presentation box. The wireless presentation box performs a snapshot of the video stream and sends the image to a list of subscribers called webviewer clients, periodically. When TCP is used for video data transfer, the rate that can is allocated for video data transfer decreases as the number of webviewer clients increases, which decreases the video quality as a result. By implementing a maximum image data transfer rate to limit the rate at which images can be downloaded by the webviewer clients, better video quality can be achieved. A question that was raised is then what data rate should a system administrator set for the maximum image data transfer rate. To answer this question, we proposed a scheme that is able to determine automatically the optimum maximum image data rate to use. Results showed that with the proposed scheme, better video quality and lower image receive time, both of which affects the overall satisfaction level of the users in a desktop mirroring session, could be achieved.

\section{REFERENCES}

[1] ISO/IEC, "ISO/IEC 14496-10: Information technology - Coding of audio-visual objects - Part 10: Advanced Video Coding," 2012.

[2] ITU-T, "ITU-T Recommendation H.265 High Efficiency Video Coding," 2015.

[3] WePresent, "wePresent WiPG-2000," wePresent, pp. 1-2, 2015.

[4] E. Electronics, "ShareLink 250 Series Wireless Collaboration Gateway," Extron Electrronics, pp. 1-6, 2016.

[5] "iPerf - The TCP, UDP and SCTP network bandwidth measurement tool." [Online]. Available: https://iperf.fr/.

[6] "The Network Simulator - ns-2." [Online]. Available: http://www.isi.edu/nsnam/ns/. [Accessed: 14-Nov-2012].

Gin-Xian Kok received his Bachelor of Engineering in ElectricalMechatronics from Universiti Teknologi Malaysia (UTM), Malaysia in 2009, and his Ph.D. in Engineering from University of Malaya (UM), Malaysia in 2015. He is currently with the Wireless Innovation Department at MIMOS Berhad, Malaysia. He has published several technical journals and conference papers.

Khong Neng Choong is a Principal Researcher at the ICT division in MIMOS Berhad, Malaysia. He is currently the head of the Collaborative Media team in the Wireless Innovation Department. He has about 12 years of working experience in the area of Information and Communication Technology. He received his M.S. and Ph.D. in Computer and Communication Systems Engineering from University Putra Malaysia, Malaysia in 1999 and 2003, respectively, and his Bachelor degree in Applied Computer Science from University of Southern Queensland, Australia in 1997. He has published over 13 technical journals and 28 conference papers, and filed over 25 patents. He has also served as cochair, committee member and technical reviewer for numerous international conferences and journals.

Danial Naghshbandi received the B.E. degree in Computer Software from Islamic Azad University of Najaf Abad, Iran in 2006 and M.S. in Computer Science from University Putra Malaysia (UPM), Malaysia in 2010. He is currently pursuing his Ph.D. in Computer Networks in UPM. $\mathrm{He}$ is also working as a Staff Researcher at wireless software development lab in MIMOS Berhad, Malaysia. His research interests include next generation mobile wireless technologies, collaborative communications, scheduling and resource allocation, and performance modeling of communication networks.

Muhammad Hilmi bin Mohd Shariff graduated from University of Manchester Institute of Science and Technology, England with B.Eng. in Computer Systems Engineering. He is currently working in MIMOS Berhad, Malaysia as a Staff Engineer in the wireless research department. With over 14 years of industrial experience, he has been developing and designing solutions and products for an array of platforms and technology. 